\title{
LOGALIZATION OF METALLOTHIONEIN IN THE BRAIN OF MACACA FASCICULARIS
}

\author{
KeIJI SUZUKI ${ }^{1}$, Katsuyuki NAKAJIMA ${ }^{2}$, UMeko KAWAHARADA ${ }^{1}$, \\ Fumiko HARA ${ }^{1}$, KatsUYoshi UEHARA ${ }^{1}$, NoRIKo OTAKI ${ }^{3}$, \\ MASAMI KIMURA ${ }^{4}$ AND YUji TAMURA ${ }^{5}$
}

Department of Pathology ${ }^{1}$, College of Medical Care and Technology, Gunma University, Showamachi 3-39-15, Maebashi 371, Japan Immunoresearch Laboratories' ${ }^{2}$, Sakaicho 17, Takasaki 370, National Institute of Industrial Health ${ }^{3}$, Tama-ku Nagao 6-12, Kawasaki, 214, Central Institute for

Experimental Animals ${ }^{4}$, Nogawa 1430, Miyamaeku, Kawasaki 213, and 1st Department of Pathology ${ }^{5}$, School of Medicine, Gunma University, Maebashi 371

Received for publication August 31, 1990 and in revised form July 5, 1991 and re-revised form May 6, 1992

\begin{abstract}
The localization of metallothionein (MT), a heavy metal binding protein of low molecular weight, in the brain of young and old macaca fascicularis was investigated by immunohistochemical technique, and the amounts of $\mathrm{MT}$ and heavy metals $(\mathrm{Zn}, \mathrm{Cu}, \mathrm{Cd})$ were measured by radioimmunoassay and atomic absorption spectrophotometer, respectively. Immunohistochemically, MT was found in pia mater, ependymal cells, protoplasmic astrocytes of the gray matter and fibrous astrocytes of the white matter of cerebrum. Cytoplasm and processes of protoplasmic and fibrous astrocytes showed strong MT immunostaining. Also, vascular feet and adventitia gave positive MT immunostaining. Moreover, both protoplasmic and fibrous astrocytes in pons and spinal cord showed the same positive MT immunostaining as those in cerebrum. In the cerebellum, Bergmann's glias, protoplasmic astrocytes of granular layer and nucleus dentatus, and fibrous astrocytes of white matter showed a strongly positive immunostain for MT.

According to the radioimmunoassay, the amount of MT in the brain of the old one was relatively highabout $19.3 \mu \mathrm{g} / \mathrm{g}$ wet weight. For the heavy metals in the brain, zinc and copper were detected, excepting cadmium.
\end{abstract}

Metallothionein (MT), an intracellular metalloprotein of low molecular weight $(6,000 \sim 7,000)$, has a high content of cystinyl residues $(33 \%)$ and a high affinity for heavy metal ions such as zinc, copper, cadmium, mercury, silver and platinum $(1,6,20)$. It is believed to play physiological roles in the storage and transport of essential metals as well as detoxication of toxic metals (25). Immunohistochemically, the location of MT has been shown not only in the liver and kidney $(2,13,23)$, but also in the various organs such as the respiratory epithelial cells of the lung (8), the surface columnar epithelial cells of the intestinal villi (7), the Paneth cells of the small intestine (8), the Sertoli and interstitial cells of the testis (8), and glandular

Mailing adress: Keiji Suzuki, M. D. Department of Pathology, College of Medical Care and Technology, Gunma University, Showamachi 3-39-15, Maebashi 371, Japan. epithelial cells of the prostate (24). In the present study, we investigated the localization of MT in the brain of macaca fascicularis by immunohistochemistry.

\section{MATERIAL AND METHODS}

Preparation of Antibody to Rat Liver MT

Preparation and validation of antibodies against MT conjugated to purified ascaris extract (10) were described in previous reports in detail $(11,15,23)$.

Rat MT-I was isolated and purified from the liver of Cd-treated rat according to the method of Tohyama and Shaikh (22). Purified rat MT-I was coupled to ascaris extract and bovine serum albumin or absorbed to polyvinyl pyrrolidon (PVP), obtaining three high molecular weight MTs and inducing high titer of MT antibodies, which subsequently were mixed with 
Freund's complete adjuvant and emulsified. The emulsions were injected subcutaneously in the backs of the New-Zealand white rabbits six times biweekly. Thereafter, the rabbits were boosted by the intravenous injections of MT-I four times biweekly. The antibody against MT conjugated to ascaris extract was absorbed with ascaris prior to use for MT immunostaining, to avoid an interference of a specific reaction to the substance of ascaris in the tissue with MT immunostaining. Its immunoreactivity to $\mathrm{MT}$ in the antiserum was confirmed by immunoblot method after sodium dodecylsulfate-polyacrylamide gel (10 20\% gradient gel) electrophoresis (SDS-PAGE); rat MT-1 protein was also detected with silver staining technique after SDS-PAGE.

\section{Histological and Immunohistochemical procedures}

The brain and spinal cord of 4 and 16 year-old female macaca fascicularis (crabeating macaque) was immediately fixed in a $10 \%$ formalin solution after autopsy. Paraffin sections of cerebrum, cerebellum, pons and spinal cord were prepared for hematoxylin eosin and immunohistochemical staining.

Immunohistochemical localization of MT was detected by anti-MT rabbit serum, using Vectastain avidin-biotinylated horseradish peroxidase complex kit (Vector Laboratories) on paraffin sections.

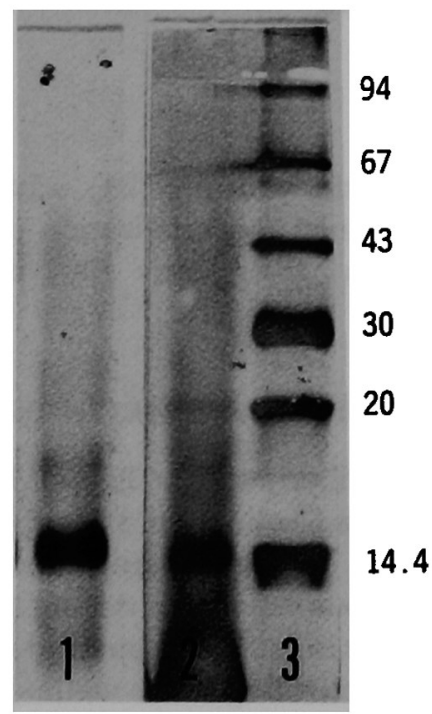

FIG. 1. Metallothionein protein (lane 2) was stained at single band by silver staining after SDS-PAGE, and after blotting onto nitrocellulose membrane, MT was stained at single band by anti-MT antibody (lane 1). Lane 3: molecular weight standards $(\mathrm{kDa})$.
Analytical Methods

MT in the cerebrum of 16 year-old macaca fascicularis was determined by essentially the same radioimmunoassay as reported earlier (11). For heavy metal analysis, the specimen of the cerebrum was digested with hot concentrated sulfuric acid, and then the contents of zinc, copper and cadmium were measured by atomic absorption spectrophotometer (Perkin Elmer 5000 Atomic Absorption Spectrophotometer).

\section{RESULTS}

Purified protein was considered pure, since no additional protein bands could be detected after staining with the silver staining technique (Fig. 1)

Immunoblotting showed antiserum against MT conjugated to ascaris extract, to be specific (Fig. 1).

\section{Absorption test}

The use of non-immune rabbit serum and

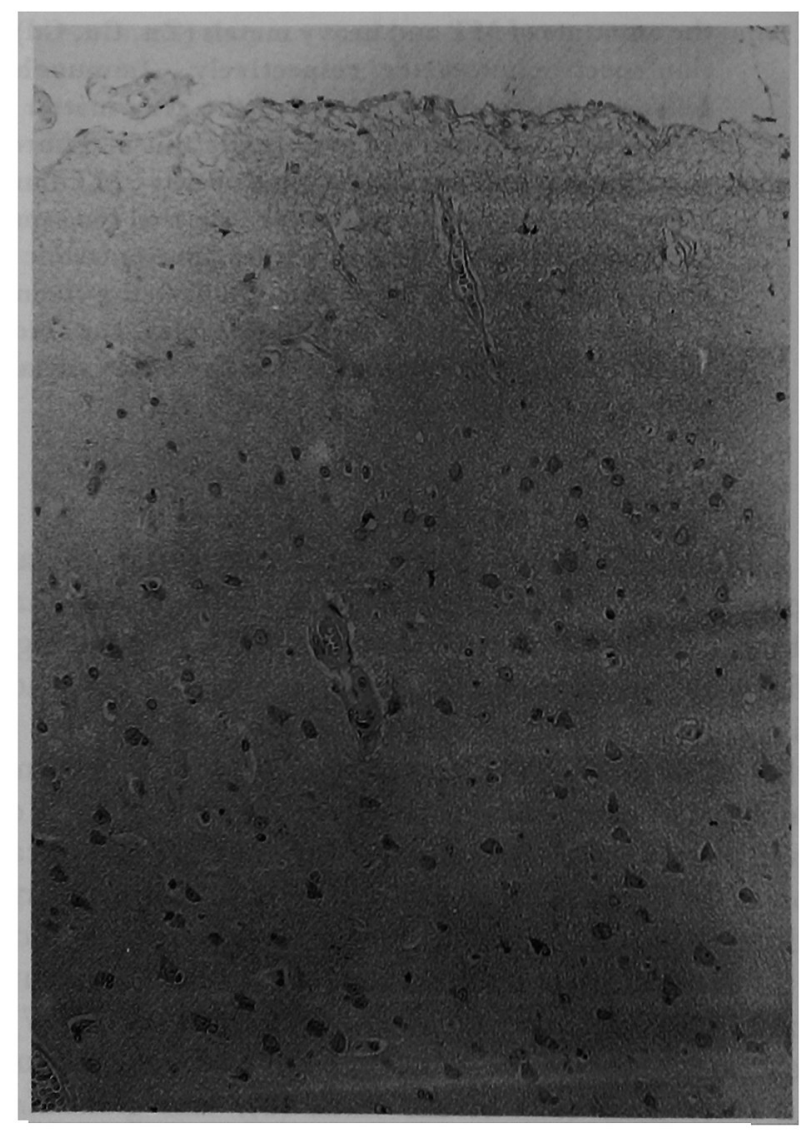

Fig. 2. Brain of macaca fascicularis shows negative MT immunostaining, using anti-MT serum conjugated with ascaris, after absorption with rabbit liver MT. ABC method. $\times 42$ 
phosphate buffered saline ( $\mathrm{pH} 7.2)$ did not result in any specific MT immunostaining in the brain of macaca fascicularis. Moreover, the three kinds of anti-MT sera that had been absorbed with rabbit liver MT (Sigma Chemical Co.) did not show a positive MT immunostain in the brain (Fig. 2).

\section{Immunohistochemical findings}

Three sera of anti-MT conjugated to ascaris or bovine serum albumin or PVP immunohistochemically showed positive for immunoreactive MT in the brain, and the antibody against MT conjugated with ascaris stained MT of the brain was the best of the three (Fig. 3).

\section{Cerebrum}

MT was found in the specific type of cells in the gray and white matter, pia mater, ependyma which lined the ventricles (Fig. 4), and epithelial layer of the choroid plexus.

In the gray matter, the cytoplasm, processes and nuclei of the protoplasmic astrocytes exhibited strong- ly positive immunostaining for MT (Fig. 5). The processes of these were found around the ganglion cells. The vascular feet of the astrocytes and adventitia of the blood vessels showed positive immunostaining (Fig. 5).

In the white matter, the processes, cytoplasm and nuclei of fibrous astrocytes showed strongly positive immunostaining for MT (Fig. 6). The immunostaining of the fibrous astrocytes was stronger than that of the protoplasmic astrocytes. The vascular feet of the astrocytes and adventitia were also strongly positive to MT immunostaining (Fig. 6).

The astrocytes in the basal ganglia and thalamus were also positive for immunostaining.

Oligodendroglia and microglia showed negative immunostaining for MT in the cerebrum.

\section{Cerebellum}

The molecular layers showed fibrillarly or granularly positive immunostaining for MT, and Bergmann's glias in the outermost area of granular layere were positive for MT (Fig. 7). Protoplasmic
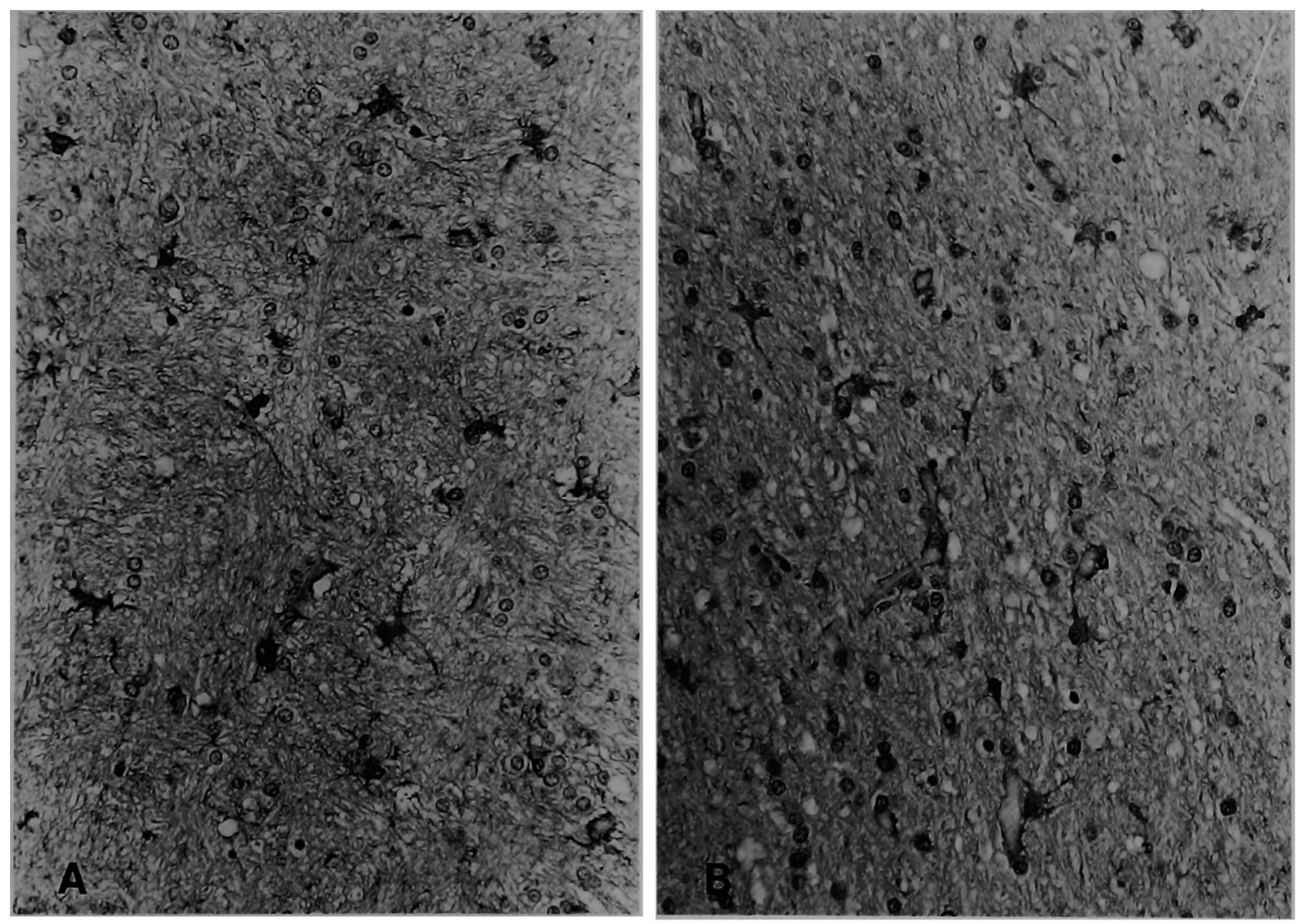

Fig. 3. Fibrous astrocytes and vascular adventitia of white matter are positive to MT immunostaining. A contrast between the astrocytes and background is not distinct. A. Stained using anti-MT serum conjugated with bovine serum albumin. B. Stained by using anti-MT serum absorbed to PVP. ABC method. $\times 107$ 
TABLE 1. Metallothionein, zinc, copper and cadmium concentration in the cerebrum of macaca fascicularis

\begin{tabular}{cccc}
\hline MT & Zn & Cu & Cd \\
\hline $19.3 \mu \mathrm{g} / \mathrm{g}$ wet wt & $17.2 \mu \mathrm{g} / \mathrm{g}$ wet wt & $6.9 \mu \mathrm{g} / \mathrm{g}$ wet wt & not detected \\
\hline wt: weight & & &
\end{tabular}

astrocytes and adventitias of blood vessels in the granular layer, and fibrous astrocytes in the white matter showed a positive MT immunostain. The protoplasmic astrocytes in the nucleus dentatus were strongly stained for immunoreactive MT.

\section{Pons}

Protoplasmic astrocytes in the nuclei pontis showed positive MT immunostaining and fibrous astrocytes in the longitudinal tract were also positive (Fig. 8). Processes of fibrous astrocytes were found around the nerve fibers of the longitudinal tract.

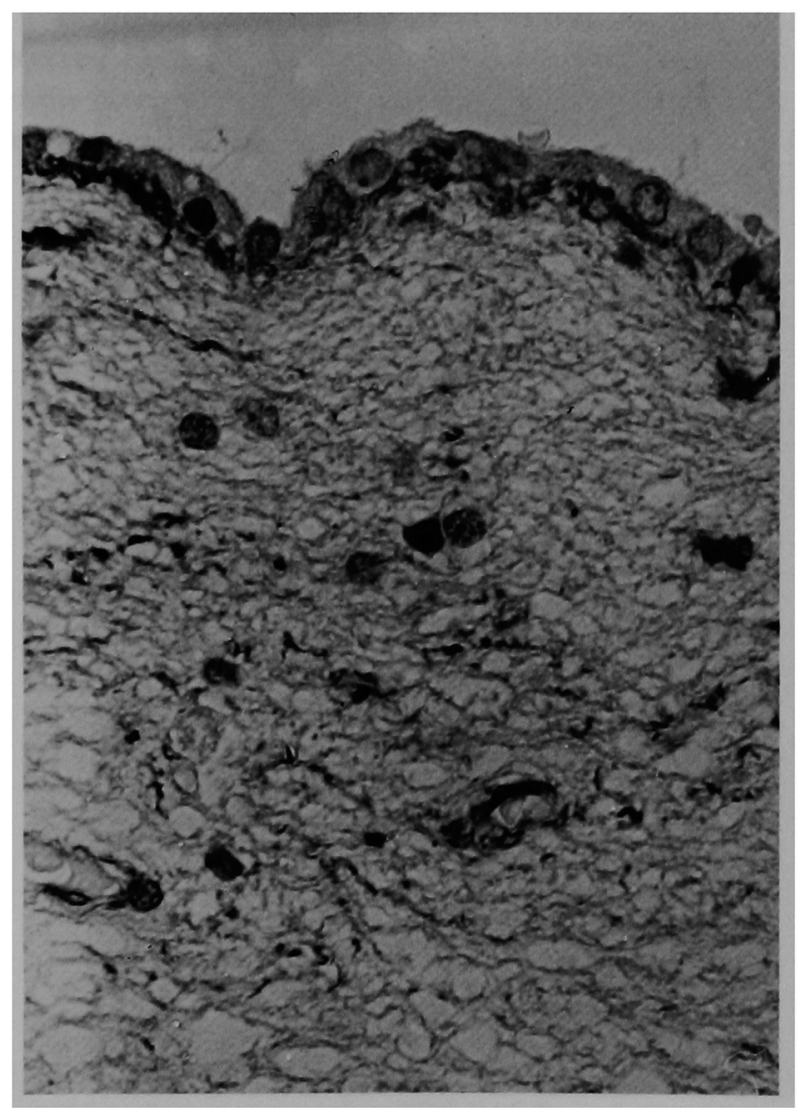

Fig. 4. Ependyma is positive for MT-immunostaining using anti-MT serum conjugated with ascaris. ABC method. $\times 214$
Spinal cord

Pia mater, astrocytes in the gray and white matter, and ependymal cells were positive for MT immunostaining.

The findings of MT immunostaining were in the similar areas of the central nervous system of 4 and 16 year-old monkeys.

\section{Analytical Findings}

Table 1 summarizes concentration of $\mathrm{MT}, \mathrm{Zn} \mathrm{Cu}$ and $\mathrm{Cd}$ in the cerebrum of macaca fascicularis.

\section{DISCUSSION}

The antiserum against MT conjugated to ascaris extract which we used in these studies was shown to be specific by immunoblotting.

The antiserum in lanes 1, 2 was indicated at the molecular weight of $14.4 \mathrm{KD}$ which are the polymerized form of MT. This is the normal characteristic of MT; low molecular weight and high in cysteine. It easily polymerizes to be dimers and higher aggregates by oxidative crosslinking (disulfide bond) (16). Furthermore, the liver, kidney and other organs containing MT and stained by this antiserum were reported to be specifically positive $(15,19,24)$.

MT was demonstrated in the rat brain (5), and MT-like protein has been identified in the rat brain (9). This study demonstrated the presence of MT by radioimmunoassay, and $\mathrm{Zn}$ and $\mathrm{Cu}$ by atomic absorption spectrophotometer in the cerebrum of 16 year-old macaca fascicularis.

Immunohistochemically, MT was localized in the protoplasmic astrocytes in the gray matter, fibrous astrocytes in the white matter, adventitia of lood vessels, pia mater, ependyma and epithelial layer of choroid plexus of the brain, but was not found in the oligodendroglias and microglias. MT was found in the nuclei, cytoplasmic process and vascular feet of both protoplasmic and fibrous astrocytes. MT may be involved in detoxification of heavy metals and may also play a role in the storage, transport, absorption, excretion and homeostasis of heavy metals in the tissues (6). 


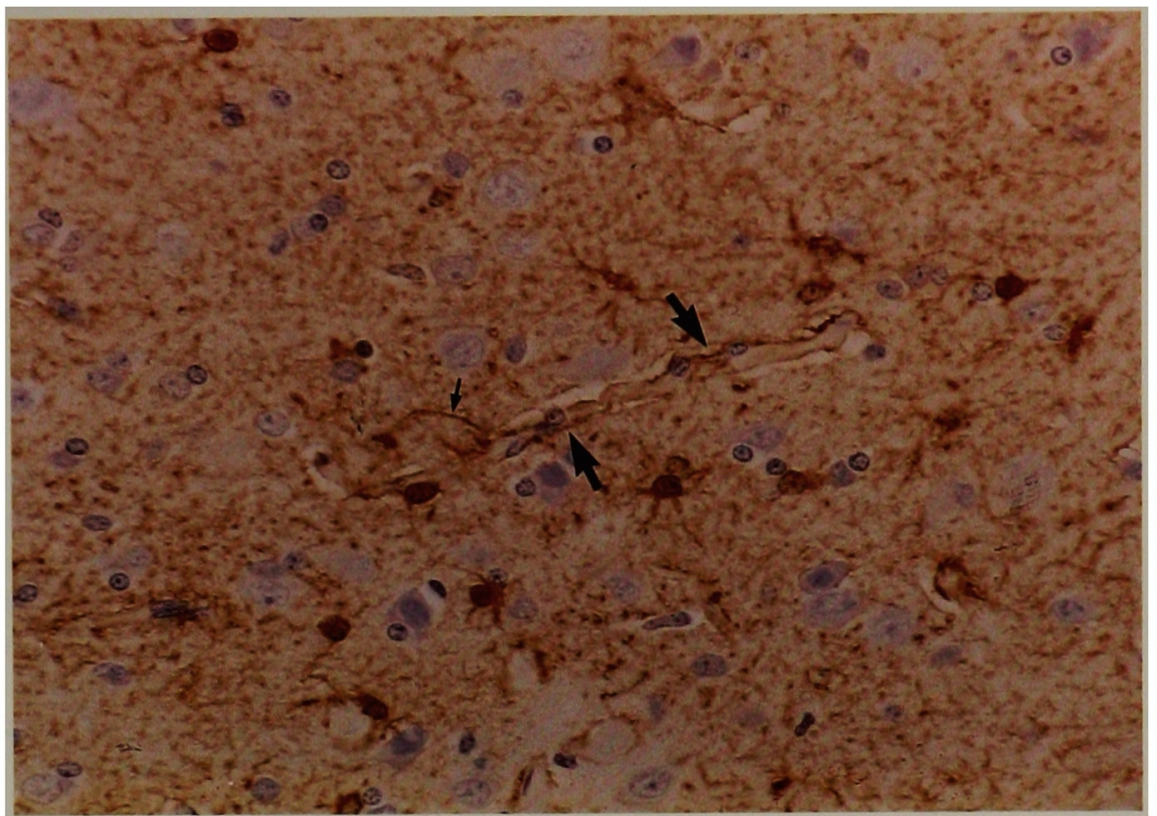

FIG. 5. The processes, cytoplasms and nuclei of protoplasmic astrocytes, vascular foot (small arrow) and blood vessel (large arrows) of gray matter are strongly positive for MT-immunostaining using anti-MT serum conjugated with ascaris. $\mathrm{ABC}$ method. $\times 214$

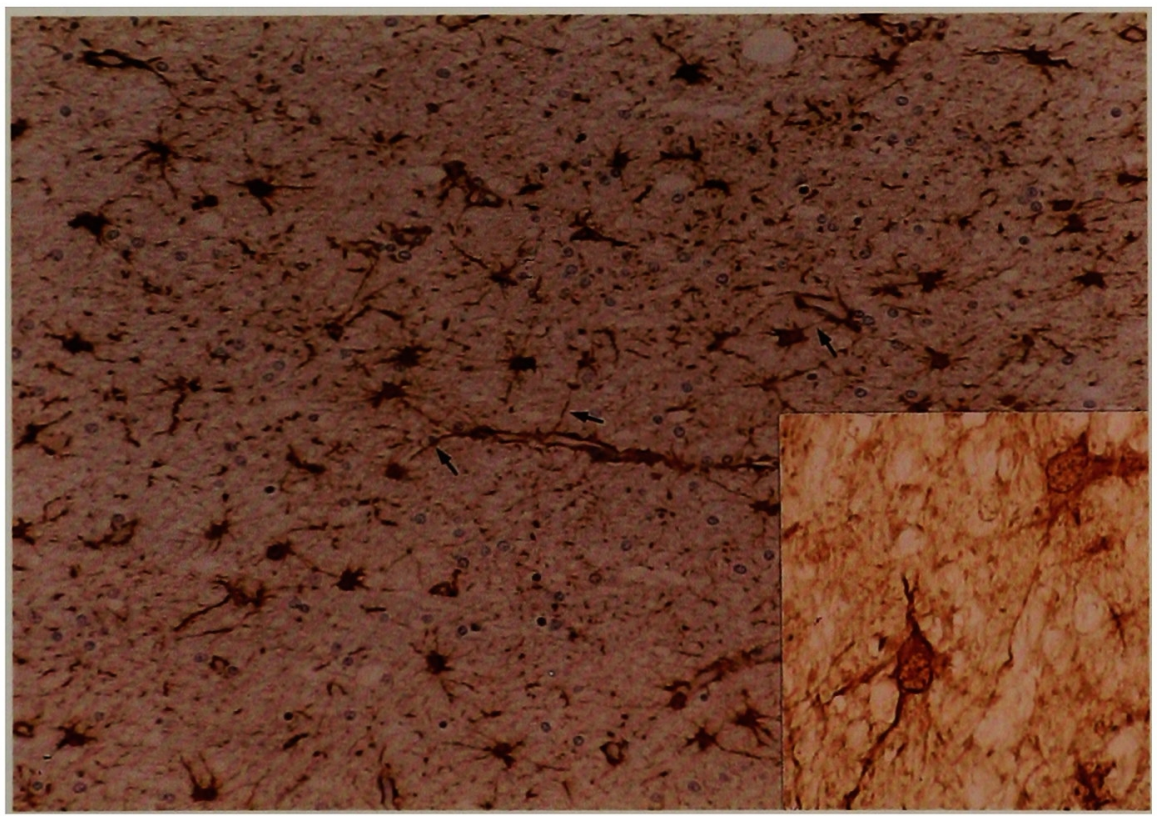

Fig. 6. The processes, cytoplasm and nuclei of fibrous astrocytes, vascular feet (arrows) and vascular adventitia of white matter are strongly positive to MT immunostaining using anti-MT serum conjugated with ascaris. ABC method. $\times 107$ Insert: Nuclei of fibrous astrocytes are positive for MT immunostain without counterstain. ABC method. $\quad \times 429$ 


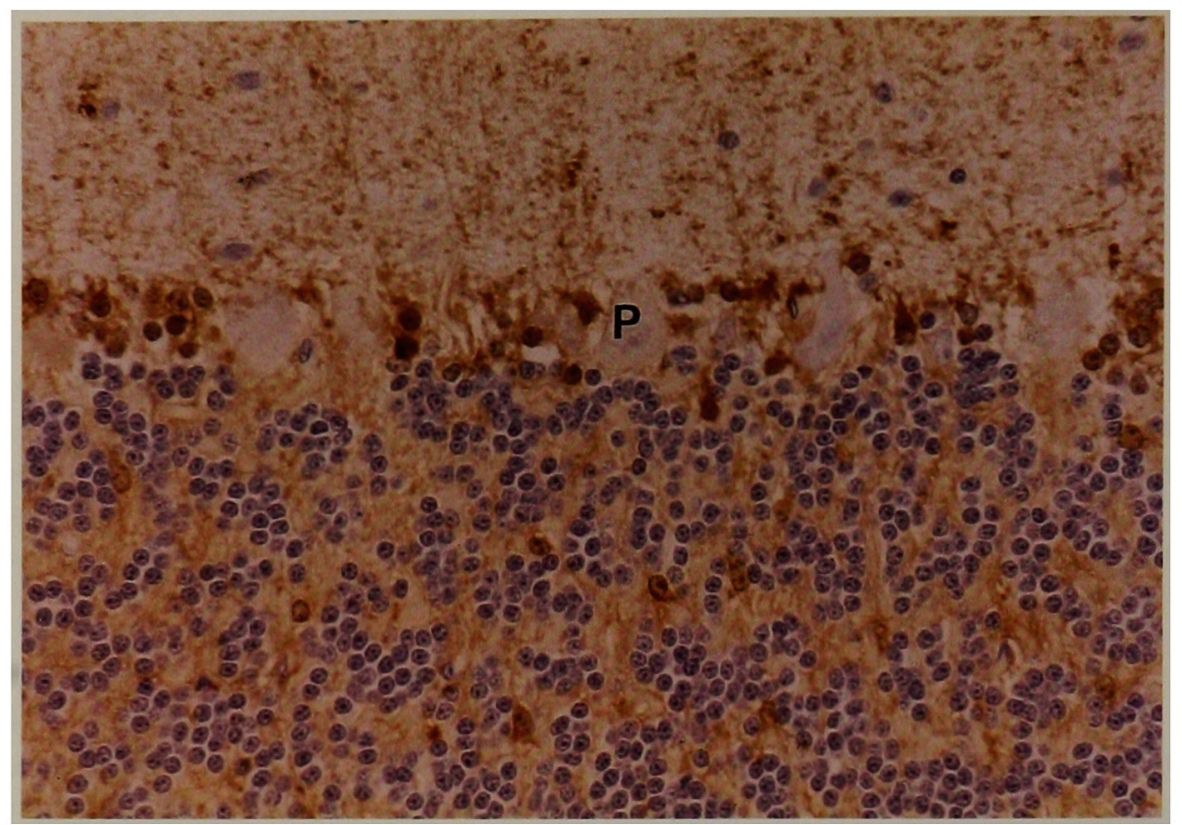

Fig. 7. Bergmann's glias and protoplasmic astrocytes in the granular layer of cerbellum are strongly positive to MT-immunostaining using anti-MT serum conjugated with ascaris. P: Purkinje cell. ABC method. $\times 214$

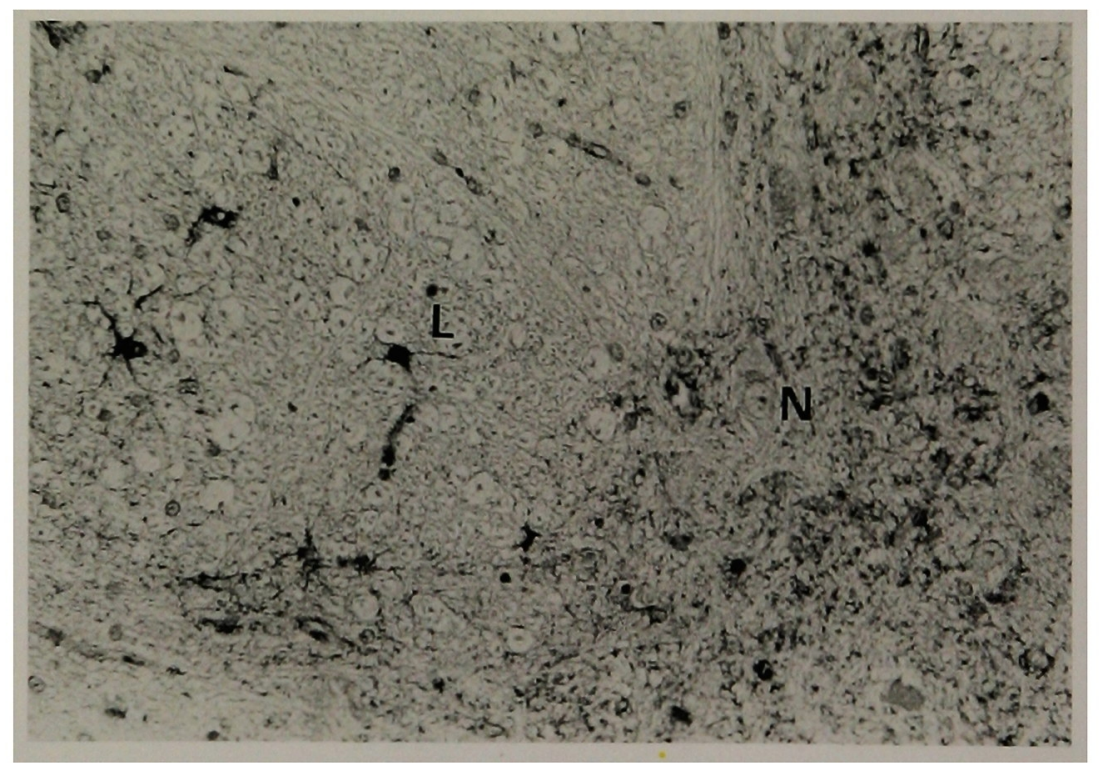

Fig. 8. Fibrous astrocytes in the longitudinal tract $(\mathrm{L})$ and processes of protoplasmic astrocytes in the nucleus pontis (N) are strongly positive to MT-immunostaining using anti-MT serum conjugated with ascaris. ABC method. $\times 214$

Zinc and copper detected in the cerebral tissue by atomic absorption spectrophotometer may be bound to some MT in the neuroglial cells. These cells seem to be an important mediator for the normal metabolism of neurons, because they have vascular feet and their processes are found around the neuron.

$\mathrm{MT}$ in the astrocytes may play a role in the transport of $\mathrm{Zn}$ and $\mathrm{Cu}$ from blood to neuron, according to this study revealing that the processes of astrocytes were found around the ganglion cells and were attached to the vascular adventitia.

Zinc has an important role in the development of central nervous system, because $\mathrm{Zn}$ defficiency induces a severe congenital malformation of the central 
nervous system in humans (4). Ohtake and Koga (18) considered that MT was important in the regulation of DNA synthesis through the synthesis or activity of enzymes required for nucleic acid. Nishimura et al. (17) have also demonstrated a possible involvement of MT in cell proliferation and differentiation. From these results, MT of the astrocytes may have an important role in the transport of $\mathrm{Zn}$ to the brain cells during the development of the central nervous system, and may play a role in the storage and homeostasis of heavy metals after its development.

Immunohistochemically, we found MT localized in nuclei of the astrocytes. The significance of the nuclear MT expression was thought to be the interaction of metal ions with various constituents of the nucleus; they might bind to histons, nucleolar RNA, and nuclear proteins (14).

MT can act as a detoxication site for reactive chemicals (12), and as an efficient free radical scavenger (21). The activities of $\mathrm{CuZn}$-superoxide dismutase were significantly decreased by $\mathrm{Zn}$ or $\mathrm{Cu}$ deficiency (3). MT in the astrocytes may have important function in the cellular defence mechanism against reactive organic intermediates and free radicals in the brain.

Pia mater showed positive immunostaining for MT. This result suggests that pia mater may have a cerebrospinal fluid-brain function against the heavy metals, because MT plays a role in detoxication of toxic metals.

Ependyma contained MT in the cytoplasm and we found the MT in the epithelial layer in the choroid plexus. The source of cerebrospinal fluid is commonly assumed to be the choroid plexus which secretes the substances in the fluid. The choroid plexus which accompany the ependyma is considered to have a function of $\mathrm{Zn}, \mathrm{Cu}$ and other heavy metal-homeostasis, and acts as a barrier function against the toxic metals in the brain.

\section{REFERENCES}

1. Andersen, R. D., Bakka, Bannister, J. V., Berger, C., Bethune, J. L., Brady, F. O., Brändén, C.-I., Bremner, I., Cherian, M. G., Cousins, R. J., Evans, G. W., Hill, H. A. O., kägi, J. H. R., Kamen, M. D., Kimura, M., Kissling, M. M., Kojima, Y., Lerch, K., Meister, A., Nordberg, G. F., Nordberg, M., Olafson, R. W., Otaki, N., Otvos, J. D., Overnell, J., Paŕízek, J., Piotrowski, J. K., Piscator, M., Pulido, P., Riordan, J. R., Rupp, H., Shaikh, Z. A., Smith, W. R. D., Vallee, B. L., Vašák, M., Webb, M., Weser, U. and Yoshida, A.: Metallothionein and other low molecular weight metal- binding protein. Metallothinein, ed. by $\mathrm{H}$. R. Kagi and M. Nordberg, Brikhauser Verlag, Basel, Boston, Stuttgart, 1979, p. 41.

2. Banerjee, D., Onosaka, S. and Cherian, M. G.: Immunohistochemical localization of metallothionein in cell nucleus and cytoplasm of rat liver and kidney. Toxicology 24; 95-105, 1982.

3. Bettger, W. J. and Bray, T. M.: Effect of dietary zinc or copper deficiency on catalase, glutathione peroxidase and superoxide dismutase activities in rat heart. Nutr. Res. 9; 319-326, 1989.

4. Cavdar, A. O., Babacan, E., Arcasoy, A. and Ertem, U.: Effect of nutrition on serum zinc concentration during pregnancy in Turkish women. Amer, J. Nutr. 33; 542-544, 1980.

5. Chen, R.W. and Ganther, H. E.: Relative cadmium binding capacity of metallothionein and other cytosolic fraction in various tissues of the rat. Environ. Physiol. Biochem. 5; 378-388, 1975.

6. Cherian, M. G. and Goyer, R. A.: Metallothioneins and their role in the metabolism and toxicity of metals. Life. Sci. 23; 1-10, 1978.

7. Clarkson, J. P., Elmes, M. E., Jasani, B. and Webb, M.: Histological demonstration of immunoreactive zinc metallothionein in liver and ileum of rat and man. Histochem. J. 17; 343-352, 1985.

8. Danielson, K. G., Ohi, S. and Huang, P. C.: Immunochemical detection of metallothinonein in specific epithelial cells of rat organs. Proc. Natl. Acad. Sci. USA 79; 2301-2304, 1982.

9. Ebadi, M. and Swanson, S.: Characterization of metallothionein-like protein in rat brain. Experientia Suppl. 52 (Metallothionein II); 289-291, 1987.

10. Hussain, R., Bradbury, S. M. and Strejan, G.: Hypersensitivity to ascaris antigens. VIII. Characaterization of highly purified allergen. J. Immunol. 111; 260268,1973

11. Ikei, N., Kodaira, T., Shimizu, F., Nakajima, K., Tohyama, C., Saitoh, H., Kimura, M. and Otaki, N.: New radioimmunoassay of metallothionein. Rinshyo Kensa $33 ; 215-219,1989$. (in Japanese)

12. Klaassen, C. D. and Cagen, S. Z.: Metallothionein as a trap for reactive organic intermediates. Adv. Exp. Med. Biol. 136-A; 633-646, 1881.

13. Kojima, Y. and Hamashima, Y.: Immunohistological studies of equine renal metallothionein. Acta Histochem. Cytochem. 11; 205-211, 1978.

14. Kontozoglou, T. E., Banerjee, D. and Cherian, G: Immunohistochemical localization of metallothionein in human testicular embryonal carcinoma cells. Virchows Arch. A. Pathol. Anat. 415; 545-549, 1989.

15. Nakajima, K., Suzuki, K., Otaki, N. and Kimura, M.: Detection of MT in brain. In "Methods in Enzymology", vol. 205, Metallobiochemistry, Part B, ed. by J. F. Riordan and B. L. Vallee, Academic Press, INC., San Diego, 1991, pp. 387-395.

16. Minkel, D. T., Poulsen, K., Wielgus, S., Shaw, C. F. and Petering, D. H.: On the sensitivity of metallothioneins to oxidation during isolation. Biochem. J. 191; 475- 
$485,1980$.

17. Nishimura, H., Nishimura, N. and Tohyama, C.: Immunohistochemical localization of metallothionein in developing rat tissue. J. Histochem. Cytochem. 37; 715722, 1989.

18. Ohtake, H. and Koga, M.: Purification and characterization of zinc-binding protein from the liver of the partially hepatectomized rat. Biochem. J. 183; 683-690, 1979.

19. Suzuki, K., Kawaharada, U., Uehara, K., Nakajima, K., Otaki, N. and Kimura, M.: Immunohistochemical studies on metallothioneins in the macaca fascicularis. $J$. Histochem. Cytochem. 38; 1024, 1990.

20. Templeton, D. M., Banerjee, D. and Cherina, M. G.: Metallothionein synthesis and localization in relation to metal storage in rat liver during gestation. Can. J. Biochem. Cell Biol. 63; 16-22, 1984.

21. Thornalley, P.J. and Vasak, M.: Possible role for metallothionein in protection against radiation-induced oxidative stress. Kinetics and mechanism of its reaction with superoxide and hydroxyl radicals. Biochem. Biophys. Acta 827; 36-44, 1985.

22. Tohyama, C. and Shaikh, Z.A.: Cross-reactivity of metallothioneins from different origins with rabbit antirat hepatic metallothionein antibody. Biochem. Biophys. Res. Commun. 84; 907-913, 1978.

23. Tohyama, C., Nishimura $H$. and Nishimura, N.: Immunohistochemical localization of metallothionein in the liver and kidney of cadmium-or zinc-treated rats. Acta Histochem. Cytochem. 21; 91-102, 1988.

24. Umeyama, T., Saruki, K., Imai, K., Yamanaka, H., Suzuki, K., Ikei, N., Kodaira, T., Nakajima, K., Saitoh, H. and Kimura, M.: Immunohistochemical demonstration in the rat prostate. The Prostate 10; 257-264, 1987.

25. Webb, M.: Metallothionein in regeneration, reproduction and development. Experientia suppl. 52 (Metallothionein II); 483-498, 1987. 